\title{
TESTES DE SUSCEPTIBILIDADE DE AGENTES DA CROMOMICOSE A 5-FLUOROCITOSINA E AO BUTIL-SIMPATOL (VASCULAT) (\$)
}

\author{
Maria Aparecida de Resende**, Luiz Gonzaga de Oliveira ** \\ Cid Ferreira Lopes $* * * *$ e Eduardo Osório Cisalpino $* * * * *$
}

\begin{abstract}
Foram realizados testes de susceptibilidade "in vitro" com várias amostras de agentes, isoladas de pacientes com cromomicose frente $\grave{a}$ 5-fluorocitosina $e$ ao butil-simpatol (Vasculat), nas concentraçóes de $0,1,1,0,5,010,0 \mathrm{mcg} / \mathrm{ml}$. Em alguns casos, em que se verificou resistência à 5-fluorocitosina, a concentração desta chegou a 100,0 $\mathrm{mcg} / \mathrm{ml}$.
\end{abstract}

O tratamento da cromomicose pela 5-fluorocitosina ( $($ ) vem sendo objeto de pesquisa de Lopes e cols. $(2,3,4)$. Em trabalhos anteriores $(1,5)$, relatam o controle micológico das observações e os primeiros testes de susceptibilidade à 5-fluorocitosina. No presente trabalho, relatamos o prosseguimento desses testes e os realizados com o butil-simpatol (Vasculat); esse meciicamento foi utilizado por Silva e Nazaré, em 1971 (6) no tratamento da micose em apreço, o qual lhes teria proporcionado bons resultados. Resolvemos, por isso, testá-lo também com espécies causadoras dessa doença, isoladas no referido ensaio.

\section{MATERIAL E MÉTODOS}

As amostras foram colhidas de pacientes da 2.a Clínica Dermatológica da Santa Casa de Misericórdia de Belo Horizonte, através de biopsia. Uma parte da mesma foi utilizada para estudos histológicos e outra para estudos micológicos.
As amostras foram colhidas antes e durante o tratamento com as drogas 5-fluorocitosina e butil-simpatol (Vasculat). Um fragmento da biópsia era transferido para o meio de sabouraud contendo antibiótico para o isolamento do fungo. Uma vez isolada, a amostra era repicada para o meio de Smith, onde os fungos sofriam uma adaptação, pois os testes foram realizados nesse meio.

Fazia-se então o teste de susceptibilidade semeando suspensão das amostras em placas de Petri contendo o meio de cultura incorporado com a droga 5-fluorocitosina nas concentrações finais de 0,1 , $1,0,5,0$ e $10,0 \mathrm{mcg} / \mathrm{ml}$. Os mesmos testes foram feitos também com algumas das amostras em placas de Petri, contendo o meio de Smith, incorporado com o butil-simpatol nas mesmas concentrações finais. As placas eram incubada ratura ambiente e a leitura era feita após uma semana de crescimento. Na leitura

* Trabalho realizado no Departamento de Microbiologia do Instituto de Ciências Biológicas da Universidade Federal de Minas Gerais, durante a gestão do diretor Prof. Eduardo Osório Cisalpino.

* Aluna do Curso de Pós-graduacão em Microbiologia e Imunologia do Inst de Ciências Biológicas da UFMG - Prof. Auxiliar de Ensino da Faculdade de Farmácia da UFoP e Prof. Assistente da Faculdade de Ciências Médicas de Minas Gerais.

** Prof. Assistente de Microbiologia do Inst. de Ciências Biológicas da UFMG e Prof. Titular de Microbiologia da Faculdade de Ciênciaś Médicas de Minas Gerais

*** Prof. Adjunto de Dermatologia da Faculdade de Medicina da UFMG e Chefe de Clínica Dermatológica da Santa Casa de Belo Horizonte.

**** Prof. Titular de Microbiologia do Inst. de Ciências Biológicas da UFMG e Reitor da UFMG.

(\$) Fabricada por F. Hoffmann-La Roche \& Co, de Basiléia, Suíça. Recebido para publicação em 30-1-75. 
dos resultados, as placas-controle (placas que não continham as drogas) eram classificadas com um crescimento de quatro cruzes. A leitura dos resultados das outras placas foi feita por comparação com o crescimento das placas-controle e o resultado oscilava entre quatro cruzes até valor negativo.

Algumas amostras que apresentaram resistência à 5 -fluorocitosina até $10,0 \mathrm{mcg}$ / $\mathrm{ml}$ foram também testadas com concentraçōes maiores da droga, tendo-se verificado a sua resistência até $100,0 \mathrm{mcg} / \mathrm{ml}$.

\section{RESULTADOS}

Estão apresentados nos quadros I e II.

\section{COMENTÁRIOS}

Verificou-se que a 5-fluorocitosina tem ação fungistática sobre o agente etiológico da cromomicose nas concentraçōes de 0,1 , $1,0,5,0$ e $10,0 \mathrm{mcg} / \mathrm{ml}$. Os teste mostram $_{\mathrm{S}}$ tambśm a possibilidade do aparecimento do fenômeno da resistência do fungo à droga "in vitro". Das amostras testadas à 5-fluorocitosina, tivemos:

1. Susceptíveis:

Cladosporium $s p-2$ amostras

Fonsecaea pedrosoi - 7 amostras

2. Resistentes:

Fonsecaea pedrosoi - 6 amostras Acrotheca aquaspersa Borelli, 1972 1 amostra

Cumpre ressaltar ainda que a resistência "in vitro" de agentes da cromomicose à 5-fluorocitosina foi descrita em primeira mão por nós (5) e agora no presente trabalho, tendo sido igualmente citada na clínica pela primeira vez por Lopes e cols. ( 2 e 3).

Nos testes com o butil-simpatol nenhuma das amostras examinadas mostrou susceptibilidade, tendo-se trabalhado com seis amostras de Fonsecaea pedrosoi e uma de Cladosporium sp.

\section{SUMMARY}

The sensitivity of strains isolated from patients with chromomycosis was tested in relation to 5-fluorocitosine and butyl-simpatol (Vasculat); some strains were resistant to fluorocitosine, even in the concentration of $100 \mathrm{mcg} / \mathrm{ml}$ and all of them showed complete resistance to butyl-simpatol.

\section{REFERENCIAS BIBLIOGRĀFICAS}

1. CISALPINO, E.O., RESENDE, M.A., PEIXOTO, Y., OLIVEIRA, L.G. \& LOPES, C.F. - Controle sorológico e micológico de doentes de cromomicose tratados pela 5-fluororitosina - Apresentado ao XXVI Congresso Brasileiro de Dermatologia, em Recife, PE, de 18 a 23 de outubro de 1969 .

2. LOPES, CID F., ALVARENGA, R.J., CISALPINO, E.O., MARTINELLI, B., SANTOS, P.U. \& ARMOND, S. - Tratamento da cromomicose pela 5-fluorocitosina - Primeiros resultados O Hospital, 75: 1335-1342, 1969.

3. LOPES, CID F., CISALPINO, E.O., ALVARENGA, R.J., ARMOND, S.. PORTO, R.V., MAIA, F.A.A. \& PEIXOTO, $\mathrm{Y}$. - Treatment of chromomycosis with 5-fluorocitosine - Int. J. Derm. 10: 182-191, 1971.
4. LOPES, CID F., ALVARENGA, R.J. CISALPINO, E.O., MAIA, F.A.A., RESENDE, M.A., \& OLIVEIRA, L.G. Resultados do tratamento da cromomicose pela 5-fluorocitosina - Terceira comunicação — Memória - VII Congresso Íbero-Latino-Americano de Dermalotogia, Caracas, Venezuela, de 12 a 16 de dezembro de 1971, pp. 1043-1048 .

5. OLIVEIRA, L.G., RESENDE, M.A., CISALPINO. E.O.. PEIXOTO. Y. \& LOPES, C.P. - Testes de susceptibili. lidade à 5-fluorocitosina com amostras isoladas de portadores de cromomicose em fase de tratamento. No prelo.

6. STLVA, D. \& NAZARÉ. I.P. - Butil-simpatol no tratamento da micose de I $\rightarrow$ ne e Pedroso - Rev. da Univ. Fed. do Pará, 1: 313-318, 1971. 
QUADRO I - Testes de susceptibilidade das amostras isoladas de pacientes com cromomicose tratados com a 5-flucrcito ina.

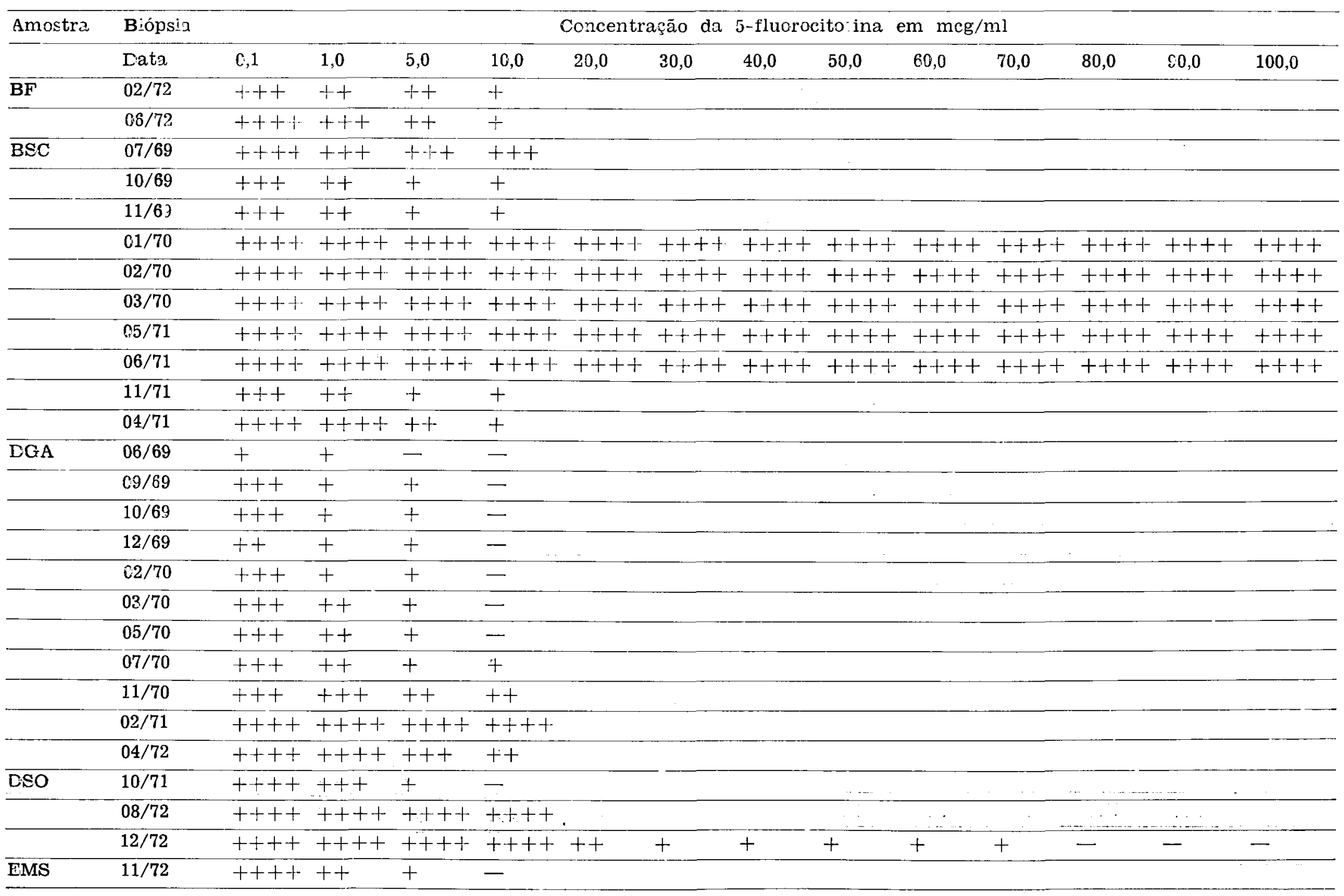


QUADRO I - Continuação.

\begin{tabular}{|c|c|c|c|c|c|c|c|c|c|c|c|c|c|c|}
\hline \multirow{2}{*}{ Amostra } & \multirow{2}{*}{$\frac{\text { Biópsia }}{\text { Data }}$} & \multicolumn{13}{|c|}{ Concentração da 5-fluorocitosina em $\mathrm{mcg} / \mathrm{ml}$} \\
\hline & & $\bar{c} 1$ & 1,0 & 5,0 & 10,0 & 20,0 & 30,0 & 40,0 & 50,0 & 60,0 & 70,0 & 80,0 & 90,0 & 100,0 \\
\hline$\overline{\mathrm{FC}}$ & $06 / 71$ & $-+\cdot++$ & ++ & + & 二 & & & & & & & & & \\
\hline \multirow[t]{7}{*}{ FS } & $05 / 69$ & + & 二 & - & - & & & & & & & & & \\
\hline & $\mathrm{C} 1 / 70$ & ++ & + & 二- & $\longrightarrow$ & & & & & & & & & \\
\hline & $07 / 70$ & $t+t$ & +++ & + & - & & & & & & & & & \\
\hline & $6.2 / 71$ & $++t+$ & $++t$ & +++ & ++ & & & & & & & & & \\
\hline & $05 / 71$ & +-+++ & -++ & $+\overline{t+}$ & ++ & & & & & & & & & \\
\hline & $06 / 71$ & $+t+$ & +++ & + & + & & & & & & & & & \\
\hline & $09 / 71$ & $+t+$ & $+t$ & ++ & - & & & & & & & & & \\
\hline \multirow[t]{3}{*}{$\mathrm{FCC}$} & $0 \% / 73$ & ++++ & +++ & + & - & & & & & & & & & \\
\hline & $11 / 73$ & $+t+t$ & +++ & + & - & & & & & & & & & \\
\hline & $04 / 74$ & $+++t$ & ++++ & ++++ & ++++ & & & & & & & & & \\
\hline \multirow[t]{4}{*}{$\mathrm{JH}$} & $01 / 72$ & +++ & ++ & - & - & & & & & & & & & \\
\hline & $04 / 72$ & +++ & +++ & + & - & & & & & & & & & \\
\hline & $07 / 73$ & ++++ & +++ & + & - & & & & & & & & & \\
\hline & $\mathrm{C} 4 / 74$ & $++t+$ & +++ & $++t+$ & ++++ & & & & & & & & & \\
\hline JPS & $02 / 72$ & +++ & +++ & + & - & & & & & & & & & \\
\hline \multirow[t]{5}{*}{$\overline{L_{I}}$} & $12 / 70$ & +++ & +++ & ++ & - & & & & & & & & & \\
\hline & $02 / 71$ & ++++ & +++ & + & - & & & & & & & & & \\
\hline & $06 / 71$ & ++++ & $+t$ & + & 一 & & & & & & & & & \\
\hline & $07 / 71$ & +++ & ++ & + & - & & & & & & & & & \\
\hline & $01 / 72$ & +++ & +++ & + & - & & & & & & & & & \\
\hline \multirow[t]{3}{*}{ MAS } & $02 / 71$ & $++t$ & +++ & + & + & & & & & & & & & \\
\hline & $05 / 72$ & ++++ & $+t+t$ & $++t$ & +++ & & & & & & & & & \\
\hline & $12 / 72$ & ++++ & ++++ & ++++ & ++++ & ++++ & ++++ & ++++ & ++++ & +++ & ++++ & ++++ & ++++ & ++++ \\
\hline \multirow[t]{4}{*}{ PDis } & $06 / 69$ & - & - & 一 & - & & & & & & & & & \\
\hline & $08 / 69$ & $+t+$ & + & 一 & - & & & & & & & & & \\
\hline & $09 / 69$ & ++++ & + & - & - & & & & & & & & & \\
\hline & $02 / 71$ & ++++ & +++ & $=$ & 二 & & & & & & & & & \\
\hline
\end{tabular}


QUADRO I - Continuação.

\begin{tabular}{|c|c|c|c|c|c|c|c|c|c|c|c|c|c|c|}
\hline \multirow[t]{2}{*}{ Amostra } & \multicolumn{5}{|c|}{ Biópsia } & \multicolumn{9}{|c|}{ Concentração da 5-fluorocitosina em $\mathrm{mcg} / \mathrm{ml}$} \\
\hline & Data & 0,1 & 1,0 & 5,0 & 10,0 & 20,0 & 30,0 & 40,0 & 50,0 & 60,0 & 70,0 & 80,0 & 90,0 & 100,0 \\
\hline \multirow[t]{2}{*}{$\overline{\mathrm{SB}}$} & $06 / 71$ & ++ & + & - & - & & & & & & & & & \\
\hline & $11 / 71$ & +++ & ++ & + & + & & & & & & & & & \\
\hline \multirow[t]{5}{*}{ VPL } & $\overline{01 / 70}$ & $+\overline{+}+$ & $+t+t$ & $++t+$ & ++++ & +++ & +++ & $++t+$ & +++ & $++t+$ & ++++ & ++++ & ++++ & +++ \\
\hline & $03 / 70$ & ++++ & ++++ & ++++ & ++++ & $++t+$ & $++t+$ & ++++ & ++++ & $+++t$ & $+++t$ & ++++ & ++++ & ++++ \\
\hline & $07 / 70$ & ++++ & ++++ & $++t+$ & ++++ & $+++t$ & $+t+t$ & $++1+$ & $++t+$ & $t+t+$ & ++++ & $++t+$ & $++t+$ & $+++t$ \\
\hline & $11 / 70$ & ++++ & +++ & +++ & $+++t$ & ++++ & $++t+$ & ++++ & ++++ & ++++ & ++++ & ++++ & ++++ & $+t+t$ \\
\hline & $09 / 72$ & $+t+t$ & ++++ & ++++ & $+++t$ & ++++ & +++1 & ++++ & ++++ & +++4 & +++ & ++++ & ++++ & ++++ \\
\hline
\end{tabular}

QUADRC II - Testes de susceptibilidade das amostras isoladas de pacientes com cromomicose tratados com o butil-simpatol (Vasculat)

\begin{tabular}{|c|c|c|c|c|}
\hline \multirow[t]{2}{*}{ Amostra } & \multirow{2}{*}{$\frac{\text { Biópsia }}{\text { Data }}$} & \multicolumn{3}{|c|}{$\begin{array}{l}\text { Concentração do butil-simpatol } \\
\text { (Vasculat) em } \mathrm{mcl} / \mathrm{ml}\end{array}$} \\
\hline & & 0,1 & 5,0 & 10,0 \\
\hline BF & $06 / 72$ & ++++++++ & +++ & ++++ \\
\hline \multirow[t]{2}{*}{$\mathrm{BSC}$} & $06 / 71$ & +++++++ & ++++ & ++++ \\
\hline & $07 / 71$ & +++++++ & $+t+t$ & $+++\dot{+}$ \\
\hline DSO & $08 / 72$ & ++++++++ & ++++ & ++++ \\
\hline $\mathrm{FC}$ & $05 / 71$ & +++++++ & ++++ & ++++ \\
\hline \multirow[t]{2}{*}{$\overline{\mathrm{JC}}$} & $09 / 71$ & ++++++++ & +++ & ++++ \\
\hline & $10 / 71$ & $+++++++t$ & ++++ & ++++ \\
\hline $\mathrm{JH}$ & $01 / 72$ & +++++++ & +++ & ++++ \\
\hline$\overline{\mathrm{LL}}$ & $02 / 71$ & ++++++++ & ++++ & ++++ \\
\hline MAS & $05 / 72$ & +++++++ & ++++ & ++++ \\
\hline
\end{tabular}

